\title{
Aspectos psicossociais do paciente com ceratocone
}

\author{
Psychological and social aspects of patients with keratoconus
}

\author{
Luciane Buggmann Moreira ${ }^{1}$ \\ João Carlos Alchieri² \\ Rubens Belfort Jr. ${ }^{3}$ \\ Hamilton Moreira ${ }^{4}$
}

\begin{tabular}{|c|}
\hline RESUMO \\
\hline 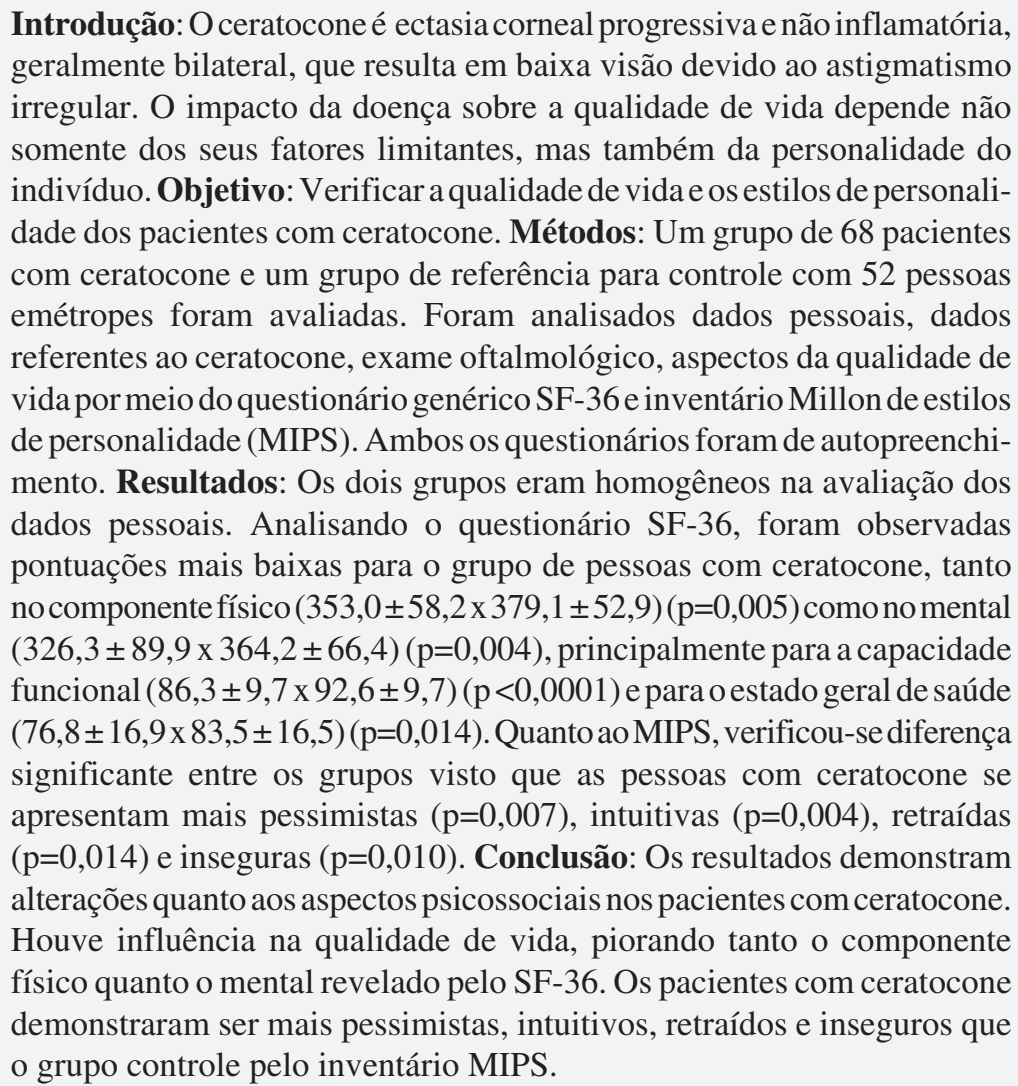 \\
\hline
\end{tabular}

Descritores: Ceratocone; Qualidade de vida; Personalidade; Questionários
Trabalho apresentado ao Departamento de Oftalmologia da UNIFESP para obtenção do título de Doutor em Ciências.

${ }^{1}$ Pós-graduanda do Programa de Doutorado em Ciências do Departamento de Oftalmologia da Universidade Federal de São Paulo - UNIFESP - São Paulo (SP) - Brasil. ${ }^{2}$ Professor Adjunto de Psicologia da Universidade Federal do Rio Grande do Norte - UFRN - Natal (RN) - Brasil.

${ }^{3}$ Professor Titular de Oftalmologia da UNIFESP - São Paulo (SP) - Brasil.

${ }^{4}$ Professor Titular de Oftalmologia da Universidade Federal do Paraná - UFPR - Curitiba (PR) - Brasil.

Endereço para correspondência: Rua Coronel Dulcídio, 199 - Térreo - Curitiba (PR) CEP 80420-170

E-mail: moreira_luciane@hotmail.com

Recebido para publicação em 23.03.2006

Última versão recebida em 07.10.2006

Aprovação em 07.11.2006

\section{INTRODUÇ̃̃̃O}

O ceratocone é caracterizado por uma ectasia não inflamatória da córnea em sua dimensão axial, usualmente tornando-se manifesto na idade jovem ou adolescência e resulta em baixa visão devido ao astigmatismo irregular miópico $^{(1)}$.

Acredita-se que algumas doenças são conseqüências de desequilíbrios emocionais e de um mau gerenciamento das emoções humanas ${ }^{(2)}$.

Da mesma forma, foi observado que pessoas com doenças crônicas mostram personalidades afins ${ }^{(3)}$.

Em 1965, Copeman já referia um comportamento ímpar em portadores de ceratocone $^{(4)}$. Desde então, vários estudos sobre ceratocone e seus efeitos psicológicos foram realizados, porém não houve concordância nos resultados dos estudos. Esses estudos tentavam identificar uma personalidade 
especifica para a doença, mas o que aparentemente se observou foram variações dentro da normalidade, por ser o ceratocone uma doença crônica ${ }^{(3,5-9)}$.

A personalidade não é somente um dado biológico transmitido pela hereditariedade. Antes de tudo, trata-se da interação da genética do indivíduo com o meio ambiente. Tal interação pode sofrer perturbações e desorganizações sob o efeito de traumatismos que provêm do exterior ou do interior do indivíduo ${ }^{(10)}$.

Concomitante à personalidade, a qualidade de vida também pode ser afetada por doenças e seus tratamentos ${ }^{(11)}$.

As doenças oculares são de grande impacto na qualidade de vida, pois a diminuição da acuidade visual (AV) é causa importante de incapacidade ${ }^{(12)}$.

Verificando a qualidade de vida, estamos avaliando a percepção que o paciente tem da sua doença e de suas repercussões sobre seu comportamento, e a personalidade tem influência direta sobre essa percepção.

Cada vez mais a medicina preocupa-se em entender os pacientes para melhor atender suas necessidades. Vários trabalhos tentam quantificar a qualidade de vida de pacientes em diversas doenças oculares, entre elas o ceratocone ${ }^{(11-14)}$.

A frequiência da doença estudada e o eventual comprometimento do psiquismo e comportamento dos pacientes, com perda visual evolutiva, estimularam o estudo da qualidade de vida e da personalidade das pessoas acometidas por ceratocone.

O objetivo deste estudo é verificar a qualidade de vida pelo questionário SF-36, os estilos de personalidade dos pacientes com ceratocone pelo MIPS e verificar se existe um fator específico agravante.

\section{MÉTODOS}

Estudo realizado de forma transversal, observacional, controlado e contemporâneo, por um único oftalmologista, no consultório particular do setor de lente de contato do Hospital de Olhos do Paraná. Foi aprovado pelo comitê de ética em pesquisa da Universidade Federal de São Paulo. Todos os participantes assinaram consentimento por escrito.

De forma aleatória e não randomizada os pacientes com ceratocone responderam aos questionários de autopreenchimento, juntamente com a realização do exame oftalmológico completo e exame topográfico. Para controle, respondeu aos mesmos questionários um grupo de voluntários emétropes. Ambos os grupos responderam às questões sobre dados pessoais, o questionário genérico SF-36, para avaliar a qualidade de vida e o inventário Millon de estilos de personalidade (MIPS), para avaliação da personalidade. Todos de autopreenchimento.

O SF-36, já traduzido e validado do inglês "Medical Outcomes Study 36-Item Short Form Health Survey", é constituído de 36 questões que abrangem os componentes físico e mental da qualidade de vida (capacidade funcional, aspectos físicos, dor, estado geral da saúde, vitalidade, aspectos sociais, aspectos emocionais e saúde mental) e também compara a percep- ção do indivíduo acerca de seu estado geral de saúde atual com a de um ano atrás ${ }^{(15)}$. Para avaliação de seus resultados, cada fator é analisado separadamente. Os dados brutos obtidos nas respostas são convertidos em dados ponderados, que são transformados em valores crescentes de 0 a 100 . O componente físico da qualidade de vida é o somatório da pontuação total da capacidade funcional, aspectos físicos, dor e estado geral da saúde. Refere-se à maneira pela qual o indivíduo interage com as sensações físicas decorrentes da sua doença. O componente mental da qualidade de vida é o somatório da pontuação total de saúde mental, aspectos emocionais, aspectos sociais e vitalidade. Refere-se à percepção de como a doença alterou o estado emocional do indivíduo ${ }^{(15)}$.

O MIPS aborda situações que as pessoas comumente experimentam, evidenciando a maneira de perceber, sentir e agir perante o mundo que as rodeia. $\mathrm{O}$ inventário abrange três áreas da personalidade: metas motivacionais, modos cognitivos e relações interpessoais, mostrando estilos de funcionamento do sujeito. Este instrumento de avaliação procura descrever em 12 bipolaridades a dominância de um ou outro estilo de personalidade para melhor equilíbrio na adaptação a vida ${ }^{(16)}$.

O MIPS, já traduzido e validado do inglês "Millon Index of Personality Styles", é composto de 180 itens de escolha verdadeiro ou falso. A avaliação dos resultados é feita por um programa de computador que consiste em analisar cada assertiva separadamente, dando valor ponderal aos componentes relacionados a ela. As 12 bipolaridades são as seguintes: 1 . abertura (otimistas) X preservação (pessimistas); 2. modificação (ativas) X acomodação (passivas); 3. individualismo (egoístas) X proteção (altruístas); 4. extroversão (expansivas) X introversão (acanhadas); 5. sensação (sensatas) X intuição (intuitivas); 6. reflexão (racionais) $\mathrm{X}$ afetividade (sentimentais); 7. sistematização (sistemáticas) $\mathrm{X}$ inovação (inovadoras); 8. retraimento (retraídas) X comunicabilidade (comunicativas); 9. vacilação (inseguras) $\mathrm{X}$ segurança (seguras); 10. discrepância (discordantes) X conformismo (conformistas); 11. submissão (submissas) $X$ controle (dominadoras); 12. insatisfação (insatisfeitas) X concordância (coerentes) ${ }^{(16)}$.

Além dos questionários mencionados, o grupo de pessoas com ceratocone responderam a dados referentes ao ceratocone. Perguntou-se sobre dificuldades específicas que o ceratocone pode causar; história familiar de ceratocone; idade do diagnóstico de ceratocone; tempo desde que a pessoa teve conhecimento de sua doença; tipo de correção mais usada. Se a resposta anterior fosse lentes de contato (LC), perguntou-se sobre conforto e horas de uso.

Essas respostas foram cruzadas com os dados obtidos dos questionários de qualidade de vida e personalidade, para verificar se existe fator específico agravante.

Como critério de inclusão, o grupo ceratocone devia ter mais de 16 anos de idade; diagnóstico de ceratocone por mais de 3 meses; não ter se submetido a transplante de córnea nem a qualquer outra cirurgia oftalmológica; não estar registrado na espera de transplante de córnea e não ter outras doenças 
oculares associadas. O grupo controle devia ter a mesma idade que o grupo em estudo e não estar em tratamento de nenhuma doença ocular nem psicológica.

Para a comprovação do objetivo proposto neste trabalho, utilizou-se a Análise de Variância (ANOVA One Way), o teste paramétrico "t de Student" e os testes não-paramétricos "Mann-Whitney" e "Kruskal-Wallis" (por meio do software "Primer of Biostatistics") e "Qui-Quadrado" (pelo Epi-Info). O nível de significância (probabilidade de significância) adotado foi menor que $5 \%(\mathrm{p}<0,05)$.

\section{RESULTADOS}

Foram avaliadas 120 pessoas, 68 portadores de ceratocone e 52 emétropes para controle.

Dos 120 participantes, $59(49,2 \%)$ eram do sexo masculino e $61(50,8 \%)$ eram do sexo feminino. A idade teve uma mediana de 25 anos. Em relação a escolaridade, 40,8\% cursaram o nível superior; $51,7 \%$ o nível médio e 7,5\% o nível fundamental. Não houve diferença estatística entre os grupos com relação aos dados pessoais já citados.

$\mathrm{Na}$ avaliação do SF-36 entre os grupos de estudo (Gráfico 1), foi observado que o grupo ceratocone apresentou menor pontuação na Capacidade Funcional $(86,3 \pm 9,7$ x 92,6 $\pm 9,7)$ $(\mathrm{p}<0,0001)$ e no Estado Geral de Saúde (76,8 $\pm 16,9$ x 83,5 \pm 16,5) $(\mathrm{p}=0,014)$.

Quando avaliados os resultados de forma genérica, dividindo em componente físico e componente mental da qualidade de vida, foram observadas pontuações mais baixas para o grupo ceratocone, tanto no Físico $(353,0 \pm 58,2 \times 379,1 \pm 52,9)(\mathrm{p}=0,005)$ quanto no Mental $(326,3 \pm 89,9 \times 364,2 \pm 66,4)(\mathrm{p}=0,004)$.

Na classificação da saúde atual, quando comparada com a de um ano atrás, observa-se no grupo controle predomínio da resposta "quase a mesma" $(67,3 \%)(\mathrm{p}=0,003)$ (Gráfico 2).

Com relação ao estudo da personalidade, o quadro 1 apresenta esquematicamente as áreas de personalidade e as respectivas 12 bipolaridades que compõem o MIPS, seguidas da quantidade de pessoas portadoras de ceratocone ( $\mathrm{G}$ cone) e emétropes ( $\mathrm{G}$ ctrl) com o estilo de personalidade que as caracterizam.

Por meio da análise comparativa dos grupos, foi observado que, na bipolaridade 1, os pacientes do grupo ceratocone possuem o estilo de personalidade de preservação $(29,4 \%)$, enquanto os pacientes do grupo controle apresentaram abertura $(92,3 \%)(\mathrm{p}=0,007)$; na bipolaridade 5 , os pacientes do grupo ceratocone apresentaram intuição $(64,7 \%)$, enquanto os pacientes do grupo controle demonstraram sensação $(63,5 \%)$ $(\mathrm{p}=0,004)$; na bipolaridade 8 , os pacientes do grupo ceratocone estão mais para retraimento $(17,6 \%)$, enquanto os pacientes do grupo controle estão mais para comunicabilidade $(98,1 \%)$ $(\mathrm{p}=0,014)$; na bipolaridade 9 , os pacientes do grupo ceratocone estão mais para vacilação $(22,1 \%)$, enquanto os pacientes do grupo controle estão mais para segurança $(77,9 \%)(\mathrm{p}=0,010)$.

Encontraram-se 19 pacientes do grupo ceratocone (28\%) com familiares acometidos e estes apresentaram pior pontua-

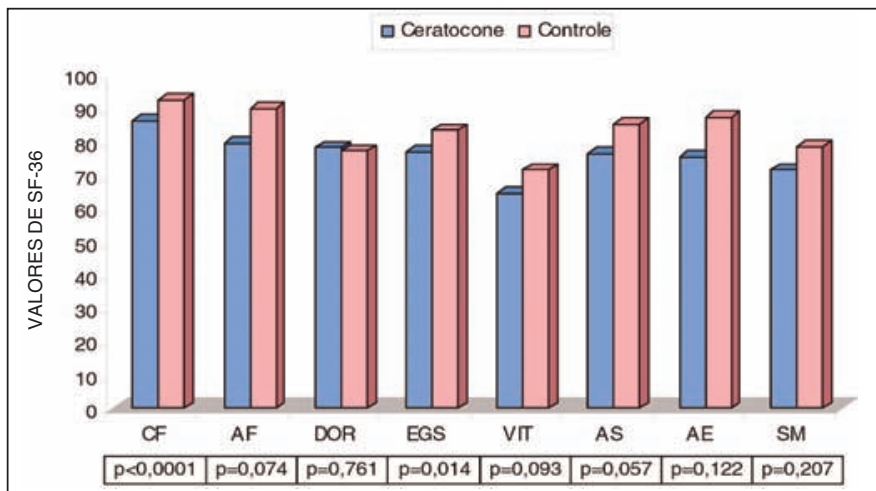

$\mathrm{CF}=$ capacidade funcional; $\mathrm{AF}=$ aspectos físicos; $\mathrm{DOR}=$ dor; $\mathrm{EGS}=$ estado geral da saúde; $\mathrm{VIT}=$ vitalidade; $\mathrm{AS}=$ aspectos sociais; $\mathrm{AE}=$ aspectos emocionais; $\mathrm{SM}=$ saúde mental

Gráfico 1 - Avaliação do questionário SF-36 em relação aos grupos de estudo

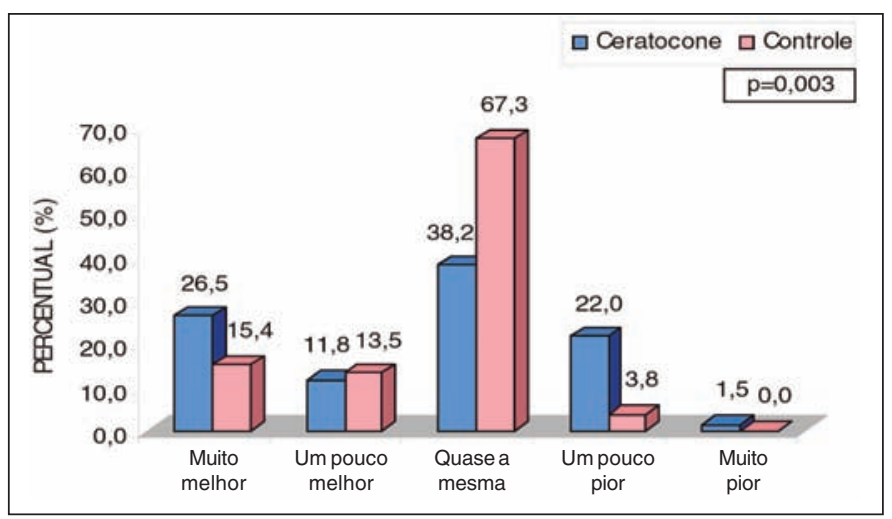

Gráfico 2 - Classificação da saúde atual comparada a de um ano atrás em relação aos grupos de estudo

ção no componente mental do SF-36 ( $\mathrm{p}=0,023)$, quando comparados aos pacientes com ceratocone sem história familiar.

A média de idade de diagnóstico foi de 21,4 anos, enquanto $45(66 \%)$ tiveram seu diagnóstico antes dos 22 anos, na adolescência. Na avaliação do MIPS foi observado que as pessoas com diagnóstico na adolescência são mais sentimentais $-6 B(p=0,042)$.

Quanto ao tempo desde que a pessoa teve conhecimento de sua doença, evidenciou-se que 14 sabiam há mais de 10 anos; 14 sabiam de seu diagnóstico entre 5 e 9 anos; 21 sabiam entre 2 e 4 anos; e 19 há menos de 1 ano. Verificou-se que conforme o tempo do diagnóstico aumenta, a classificação da saúde atual tende a melhorar.

Dos pacientes com ceratocone, $67 \%$ usavam lentes de contato. Comparando no MIPS esses pacientes com aqueles que não usam lentes, observamos nos primeiros pessoas mais otimistas $(\mathrm{p}=0,006)$, mais ativas $(\mathrm{p}=0,012)$, mais extrovertidas $(\mathrm{p}=0,027)$, mais sistemáticas $(\mathrm{p}=0,015)$ e mais comunicativas $(\mathrm{p}=0,003)$.

Trinta e um pacientes $(45,6 \%)$ com ceratocone responderam que sentiam dificuldades causadas pelo ceratocone. A interferência foi no geral para 14 pessoas $(45,2 \%)$. Para $29 \%$ das 


\begin{tabular}{|c|c|c|c|c|c|c|}
\hline & \multicolumn{6}{|c|}{ Quadro 1. Análise descritiva das metas motivacionais, dos modos cognitivos e das relações interpessoais } \\
\hline & \multicolumn{2}{|c|}{ Metas motivacionais } & \multicolumn{2}{|c|}{ Modos cognitivos } & \multicolumn{2}{|c|}{ Relações interpessoais } \\
\hline $\begin{array}{l}\text { G cone } \\
G \text { ctrl }\end{array}$ & $\begin{array}{c}1 \mathrm{~A}=\text { Abertura } \\
48(70,6 \%) \\
48(92,3 \%) \\
p=0,007\end{array}$ & $\begin{array}{c}\text { 1B = Preservação } \\
20(29,4 \%) \\
4(7,7 \%)\end{array}$ & $\begin{array}{c}A=\text { Extroversão } \\
55(80,9 \%) \\
49(94,2 \%) \\
p=0,063\end{array}$ & $\begin{array}{c}\text { 4B = Introversão } \\
13(19,1 \%) \\
3(5,8 \%)\end{array}$ & $\begin{array}{c}\mathrm{AA}=\text { Retraimento } \\
12(17,6 \%) \\
1(1,9 \%) \\
p=0,014\end{array}$ & $\begin{aligned} 8 \mathrm{~B}= & \text { Comunicabilidade } \\
& 56(82,4 \%) \\
& 51(98,1 \%)\end{aligned}$ \\
\hline $\begin{array}{l}\text { G cone } \\
G \text { ctrl }\end{array}$ & $\begin{array}{c}2 A=\text { Modificação } \\
52(76,5 \%) \\
46(88,5 \%) \\
p=0,149\end{array}$ & $\begin{array}{c}\text { 2B = Acomodação } \\
16(23,5 \%) \\
6(11,5 \%)\end{array}$ & $\begin{array}{c}5 A=\text { Sensação } \\
24(35,3 \%) \\
33(63,5 \%) \\
p=0,004\end{array}$ & $\begin{array}{c}\text { 5B = Intuição } \\
44(64,7 \%) \\
19(36,5 \%)\end{array}$ & $\begin{array}{c}9 A=\text { Vacilação } \\
15(22,1 \%) \\
2(3,8 \%) \\
p=0,010\end{array}$ & $\begin{array}{c}9 B=\text { Segurança } \\
53(77,9 \%) \\
50(96,2 \%)\end{array}$ \\
\hline $\begin{array}{l}\text { G cone } \\
\mathrm{G} \text { ctrl }\end{array}$ & $\begin{aligned} 3 A= & \text { Individualismo } \\
& 7(10,3 \%) \\
& 9(17,3 \%) \\
& p=0,396\end{aligned}$ & $\begin{array}{c}\text { 3B = Proteção } \\
61(89,7 \%) \\
43(82,7 \%)\end{array}$ & $\begin{array}{c}6 A=\text { Reflexão } \\
21(30,9 \%) \\
17(32,7 \%) \\
p=0,989\end{array}$ & $\begin{array}{c}6 \mathrm{~B}=\text { Afetividade } \\
47(69,1 \%) \\
35(67,3 \%)\end{array}$ & $\begin{array}{c}10 A=\text { Discrepância } \\
4(5,9 \%) \\
0(0 \%) \\
p=0,099^{(1)}\end{array}$ & $\begin{array}{c}10 \mathrm{~B}=\text { Conformismo } \\
64(94,1 \%) \\
52(100 \%)\end{array}$ \\
\hline $\begin{array}{l}\text { G cone } \\
\text { G ctrl }\end{array}$ & & & $\begin{array}{c}7 A=\text { Sistematização } \\
57(83,8 \%) \\
47(90,4 \%) \\
p=0,437\end{array}$ & $\begin{array}{c}7 \mathrm{~B}=\text { Inovação } \\
11(16,2 \%) \\
5(9,6 \%)\end{array}$ & $\begin{array}{c}11 \mathrm{~A}=\text { Submissão } \\
68(100 \%) \\
52(100 \%)\end{array}$ & $\begin{aligned} 11 \mathrm{~B}= & \text { Controle } \\
& - \\
& -\end{aligned}$ \\
\hline $\begin{array}{l}\text { G cone } \\
\text { G ctrl }\end{array}$ & & & & & $\begin{array}{c}12 \mathrm{~A}=\text { Insatisfação } \\
6(8,8 \%) \\
4(7,7 \%) \\
p=0,549\end{array}$ & $\begin{array}{c}12 \mathrm{~B}=\text { Concordância } \\
62(91,2 \%) \\
48(92,3 \%)\end{array}$ \\
\hline
\end{tabular}

pessoas analisadas (9), o que mais incomoda no ceratocone é não poder ficar sem as LC, por não enxergar sem elas e não conseguir usar óculos. A dificuldade de passar no teste de visão do Detran apareceu em 9,7\% dos casos (3). Para 2 pessoas $(6,4 \%)$ o ceratocone interferiu com sua vida social. Para 3 pessoas $(9,7 \%)$ o ceratocone atrapalhou seu futuro profissional, tendo de mudar de emprego ou ser reprovado em concurso, devido à sua visão.

Comparando os pacientes que responderam sentirem dificuldades com aqueles que responderam não ter havido, perante o SF-36 e MIPS, observou-se significativa piora no SF-36 ( $\mathrm{p}=0,0001)$, saúde atual um pouco pior que a de um ano atrás $(\mathrm{p}=0,025)$ e maior pontuação MIPS para retraimento $(\mathrm{p}=0,001)$.

\section{DISCUSSÃO}

Os grupos estudados são homogêneos e podem ser estatisticamente comparados entre si.

Este trabalho estudou pacientes portadores de ceratocone com conhecimento prévio do diagnóstico e não inscritos para transplante de córnea tentando verificar somente as alterações psicossociais decorrentes da doença e não o impacto causado pela notícia do diagnóstico ou pela espera do transplante de córnea.

Conforme alguns autores, pessoas com o mesmo diagnóstico mostram qualidades de vida diferentes, dependendo da personalidade ${ }^{(17)}$. O presente estudo avaliou a personalidade dos pacientes com ceratocone sem o intuito de diagnosticar distúrbios psiquiátricos.
A qualidade de vida sofre influência direta das doenças crônicas $^{(11)}$.

O ceratocone é uma doença crônica com duração extremamente longa e afeta pessoas durante a juventude. Por isso deficiências pequenas podem resultar em impacto desproporcional na qualidade de vida dessas pessoas. Conforme Vitale, quanto mais jovem o indivíduo inicia uma doença que piora sua visão, pior será sua qualidade de vida. Neste estudo observamos no MIPS pessoas mais sentimentais, quando o diagnóstico se dava na adolescência ${ }^{(14)}$.

Comparando os resultados deste estudo que utilizou o questionário SF-36 ao realizado anteriormente com o questionário NEI-VFQ, ambos de pontuação em valores crescentes, encontrou-se resultados parecidos para estado geral de saúde $(76,8 \times 75,5)$ e saúde mental $(73,1 \times 71,5)^{(18)}$.

Desde 1965 há relatos de que o ceratocone pode causar um comportamento ímpar nas pessoas por ele acometidas ${ }^{(4)}$.

Vários autores tentaram achar uma personalidade com atributos específicos para os portadores de ceratocone, porém não mostram resultados estatísticos conclusivos ${ }^{(3,5-7,19-21)}$.

Neste estudo, não se tentou achar alterações patológicas específicas para portadores de ceratocone e sim definir os estilos de personalidade dessas pessoas comparadas às pessoas emétropes pelo Inventário Millon de Estilos de Personalidade (MIPS).

Os achados desse estudo mostram diferenças significativas na personalidade dos grupos estudados. Pode-se dizer que os portadores de ceratocone apresentam-se mais pessimistas, intuitivos, retraídos e inseguros que os emétropes estudados. Dados parecidos aos encontrados por Giedd et al., 
que encontraram pessoas pessimistas ${ }^{(8)}$. Vários autores encontraram dados diferentes observando pessoas mais passivo-agressivas, paranóides e hipomaníacas ${ }^{(3)}$, associando ceratocone com comportamento de personalidade tipo A descrita por serem articuladas, sistemáticas e autoritárias ${ }^{(19-21)}$ e até associação com esquizofrenia ${ }^{(9)}$.

Dificilmente poder-se-á responder se os estilos de personalidade achados nessas pessoas são causa ou conseqüência de doença, pois tanto o comportamento quanto as doenças são dependentes de vários fatores. $\mathrm{O}$ meio onde vivemos e o que nos acontece exercem influência no funcionamento do indivíduo, da mesma forma que as emoções têm grande papel na evolução das doenças ${ }^{(22)}$. Pacientes que por ansiedade esfregam muito os olhos pode influenciar na evolução ou aparecimento de ceratocone e o impacto do diagnóstico de ceratocone pode, por sua vez, afetar o modo de agir e pensar dos pacientes. Os médicos devem entender como as emoções interferem na saúde para diminuir a ansiedade de seus pacientes tornando-os mais participativos e ajudando com o tratamento ${ }^{(8)}$.

Quanto maior o conhecimento da doença melhor a qualidade de vida ${ }^{(23)}$. Ao contrário disso, este estudo mostrou que os pacientes com história familiar de ceratocone, que supostamente deveriam ter mais conhecimento sobre a doença, tiveram maior comprometimento do componente mental do SF-36 que aqueles sem história familiar. Isso possivelmente é devido a experiências ruins com familiares. Nestes casos os oftalmologistas devem mencionar a evolução tanto das lentes de contato quanto das cirurgias.

Ainda no SF-36 é interessante observar que na pergunta sobre saúde atual comparada ao ano anterior, esta tende a ser melhor quando o diagnóstico da doença é mais antigo. Isso demonstra que quanto mais tempo a pessoa sabe de sua doença, melhor é sua aceitação e a adaptação ao fato de ter uma doença crônica se faz aos poucos.

A satisfação em sua visão está muito relacionada com a qualidade de vida de tal forma que há uma tese usando, como grupo controle, pacientes amétropes satisfeitos com sua correção óptica ${ }^{(24)}$. No presente estudo observou-se que, nos pacientes com ceratocone, a satisfação com a correção óptica faz com que eles sintam sua saúde atual melhor em comparação a de um ano atrás.

Chama atenção o fato de que pacientes com ceratocone que conseguem usar lentes de contato são mais otimistas, extrovertidos e comunicativos que aqueles que não conseguem usá-las, sugerindo a relação entre personalidade e satisfação com a correção óptica.

Alguns estudos mostram acuidade visual relacionada diretamente com as frustrações no ceratocone ${ }^{(14,18,25-27)}$.

Em contrapartida, já foi verificado que existe fraca correlação entre a pontuação na qualidade de vida e a $\mathrm{AV}^{(17)}$, da mesma forma que neste estudo não se achou correlação significante entre as pontuações do questionário SF-36 e AV ou ceratometria. Contrariamente a isso, na pergunta sobre as dificuldades que o ceratocone pode causar, observam-se respostas como incômodo de não poder ficar sem as LC, por não enxergar sem elas e não conseguir usar óculos, dificuldade de passar no teste de visão do Detran sem lentes, e que o ceratocone atrapalhou seu futuro profissional, tendo de mudar de emprego ou ser reprovado em concurso devido à sua visão.

No estudo CLEK foi verificado que, por causa do ceratocone, $1,4 \%$ mudaram de emprego; $2,1 \%$ aposentaram-se e $11,5 \%$ foram demitidos ${ }^{(25)}$. Anteriormente já foi observado que $35,6 \%$ da população estudada tem percepção de que o ceratocone afetava muito sua capacidade para trabalhar ${ }^{(12)}$. No estudo agora analisado, esta pergunta não foi específica à vida profissional, mesmo assim, 3 pessoas disseram ter mudado de emprego por causa do ceratocone.

\section{CONCLUSÕES}

A qualidade de vida é influenciada pelo ceratocone, que piora tanto o componente físico quanto o mental, revelado pelo questionário genérico SF-36.

Os pacientes com ceratocone têm estilo de personalidade mais pessimista, intuitivo, retraído e inseguro que os do grupo controle.

Todos os fatores estudados referentes ao ceratocone influenciam na qualidade de vida e personalidade das pessoas.

$\mathrm{O}$ bom uso das lentes de contato interfere no resultado do MIPS, tornando as pessoas mais otimistas, ativas, extrovertidas, comunicativas e sistemáticas que aquelas que não usam lentes de contato.

Todo oftalmologista deve estar familiarizado ao modo como a psicologia individual e o meio sociocultural afetam a condição médica, para desta forma, o diagnóstico do ceratocone e informações sobre a evolução, não criar trauma nem mal entendido no paciente.

\section{ABSTRACT}

Introduction: Keratoconus is a progressive and non-inflammatory ectasia of the cornea, mostly bilateral causing low vision because of the irregular astigmatism. The influence of the disease on quality of life is dependent on limiting factors and personality type. Purpose: To verify quality of life and personality type of the person with keratoconus. Methods: Sixty-eight individuals with keratoconus and 52 without the ocular disease were studied. The following data were collected: personal demographic data, keratoconus measurements and specific questions, complete ophthalmologic examination, measurement of quality of life and personality type using standardized instruments. Results: Both groups were demographically similar and comparable. SF-36 and MIPS differed between keratoconus and control patients. SF-36 scores showed that the keratoconus group had lower scores regarding the physical component $(353.0 \pm 58.2 \times 379.1 \pm 52.9)(\mathrm{p}=0.005)$ as well as the mental component $(326.3 \pm 89.9 \times 364.2 \pm 66.4)(\mathrm{p}=0.004)$, mainly functional capacity $(86.3 \pm 9.7 \times 92.6 \pm 9.7)(\mathrm{p}<0.0001)$ and 
general health $(76.8 \pm 16.9 \times 83.5 \pm 16.5)(\mathrm{p}=0.014)$. MIPS scores showed that patients with keratoconus were significantly more pain avoiding ( $\mathrm{p}=0.007)$ presented more, imaginative intuition $(\mathrm{p}=0.004)$, asocial withdrawal $(\mathrm{p}=0.014)$ and anxious hesitation $(\mathrm{p}=0.010)$ than the control group. Conclusion: The results showed psychosocial impairment in the keratoconus group. Keratoconus influenced the quality of life expressed by the SF-36. MIPS showed keratoconus patients to be more pain avoiding, with more imaginative intuition, asocial withdrawal and anxious hesitation than the control group.

Keywords: Keratoconus; Quality of life; Personality; Questionnaires

\section{REFERÊNCIAS}

1. Duke-Elder S, Leigh AG. Keratoconus: conical cornea. In: Duke-Elder S. System of ophthalmology. London: Henry Kimpton; 1965. v. 8 p.964-76.

2. Ballone GJ. Teorias da personalidade. PsiqWeb [periódico na Internet]. [cita do 2003 Jun 21]. Disponível em: http://www.psiqweb.med.br/persona/personal.html.

3. Mannis MJ, Morrison TL, Zadnik K, Holland EJ, Krachmer JH. Personality trends in keratoconus. An analysis. Arch Ophthalmol. 1987;105(6):798-800

4. Copeman PWM. Eczema and Keratoconus. Brit Med J. 1965;2(5468):977-9.

5. Karseras AG, Ruben M. Aetiology of keratoconus. Brit Med J. 1976;60(7): $522-5$.

6. Swartz NG, Cohen EJ, Scott DG, Genvert GI, Arentsen JJ, Laibson PR. Personality and keratoconus. CLAO J. 1990;16(1):62-4

7. Cooke CA, Cooper C, Dowds E, Frazer DG, Jackson AJ. Keratoconus, myopia, and personality. Cornea. 2003;22(3):239-42.

8. Giedd KK, Mannis MJ, Mitchel GL, Zadnik K. Personality in Keratoconus in a sample of patients derived from the internet. Cornea. 2005;24(3):301-7.

9. Rudisch B, D'Orio B, Compton MT. Keratoconus and psychosis. Am J Psychiatry. 2003;160(5):1011.

10. Bussab VSR. Fatores hereditários e ambientais no desenvolvimento: a adoção de uma perspectiva interacionista. Psicol Reflex Crit. 2000;13(2):233-43.

11. Nogueira R, Franca M, Lobato MG, Belfort R, Souza CB, Gomes JAP Qualidade de vida dos pacientes portadores de síndrome de Stevens-Johnson. Arq Bras Oftalmol. 2003;66(1):67-70.
12. Lima CA. Proposição e teste de um questionário de qualidade de vida em pacientes com ceratocone [dissertação]. Campinas: Faculdade de Ciências Médicas da Universidade Estadual de Campinas; 2000.

13. Wilson RM, Coleman AL, Yu F, Bing EG, Sasaki IF, Berlin K, et al. Functional status and well-being in patients with glaucoma as measured by the Medical Outcomes Study Short Form-36 questionnaire. Ophthalmology. 1998;105(11):2112-6

14. Vitale S. CLEK study reports on the quality of life. Am J Ophthalmol. 2004;138(4):637-8. Comment on: Am J Ophthalmol. 2004;138(4):527-35.

15. Ciconelli RM, Ferraz MB, Santos W, Meinão I, Quaresma MR. Tradução para língua portuguesa e validação do questionário genérico de avaliação de qualidade de vida SF-36 (Brasil SF-36). Rev Bras Reumatol. 1999;39(3):14350.

16. Alchieri JC. Modelo dos estilos de personalidade de Millon: adaptação do Inventário Millon de Estilos de Personalidade [tese]. Porto Alegre: Universidade Federal do Rio Grande do Sul; 2004.

17. Atique D, Goulart DG, Lake JC, Lima FA, Felberg S, Nishiwaki-Dantas MC. Qualidade de vida após transplante penetrante de córnea. Arq Bras Oftalmol. 2002;65(3):351-4.

18. Kymes SM, Walline JJ, Zadnik K, Gordon MO. Collaborative Longitudinal Evaluation of Keratoconus study group. Quality of life in keratoconus. Am J Ophthalmol. 2004;138(4):527-35. Comment in: Am J Ophthalmol. 2004; 138(4):637-8.

19. Thalasselis A, Taie HF, Etchepareborda J, Selim A. Keratoconus, magnesium deficiency, type A behavior, and allergy. Am J Optom Physiol Opt. 1988;65 (6):499-505.

20. Thalasselis A, Taie HF. Thalasselis' syndrome and other theories on keratoconus. J Am Optom Assoc. 1989;60(10):754-8.

21. Thalasselis A. Thalasselis syndrome and genetic theories on Keratoconus. J Am Optom Assoc. 1995;66(8):495-9.

22. Alves VLR. A comunicação diagnóstica de ceratocone e a sua influência na representação social que o paciente constrói da sua doença [tese]. São Paulo: Pontifícia Universidade Católica de São Paulo; 2003.

23. Cavalcanti FS, Freitas GG, Araújo MHQ, Leite MSS, Castro BTD. Nível de informação fornecida ao paciente sobre sua doença. Rev Bras Reumatol. 1993; 33(6):223-5.

24. Belfort R. Avaliação da qualidade visual e de vida de pacientes portadores de ametropias [tese]. São Paulo: Universidade Federal de São Paulo; 2001.

25. Zadnik K, Barr JT, Edrington TB, Everett DF, Jameson M, Mcmahon TT, et al. Baselines findings in the Collaborative Longitudinal Evaluation of Keratoconus (CLEK) Study. Invest Ophthalmol Vis Sci. 1998;39(13):2537-46.

26. CLEK Study Group. Collaborative Longitudinal Evaluation of Keratoconus Study (CLEK study). Operation Manual. Rockville; National Eye Institute;1996.

27. Mendes F, Schaumberg DA, Navon S, Steinert R, Sugar J, Holland EJ, et al. Assessment of visual function after corneal transplantation: The quality of life and psychometric assessment after corneal transplantation (Q-PACT) study. Am J Ophthalmol. 2003;134(6):785-93.

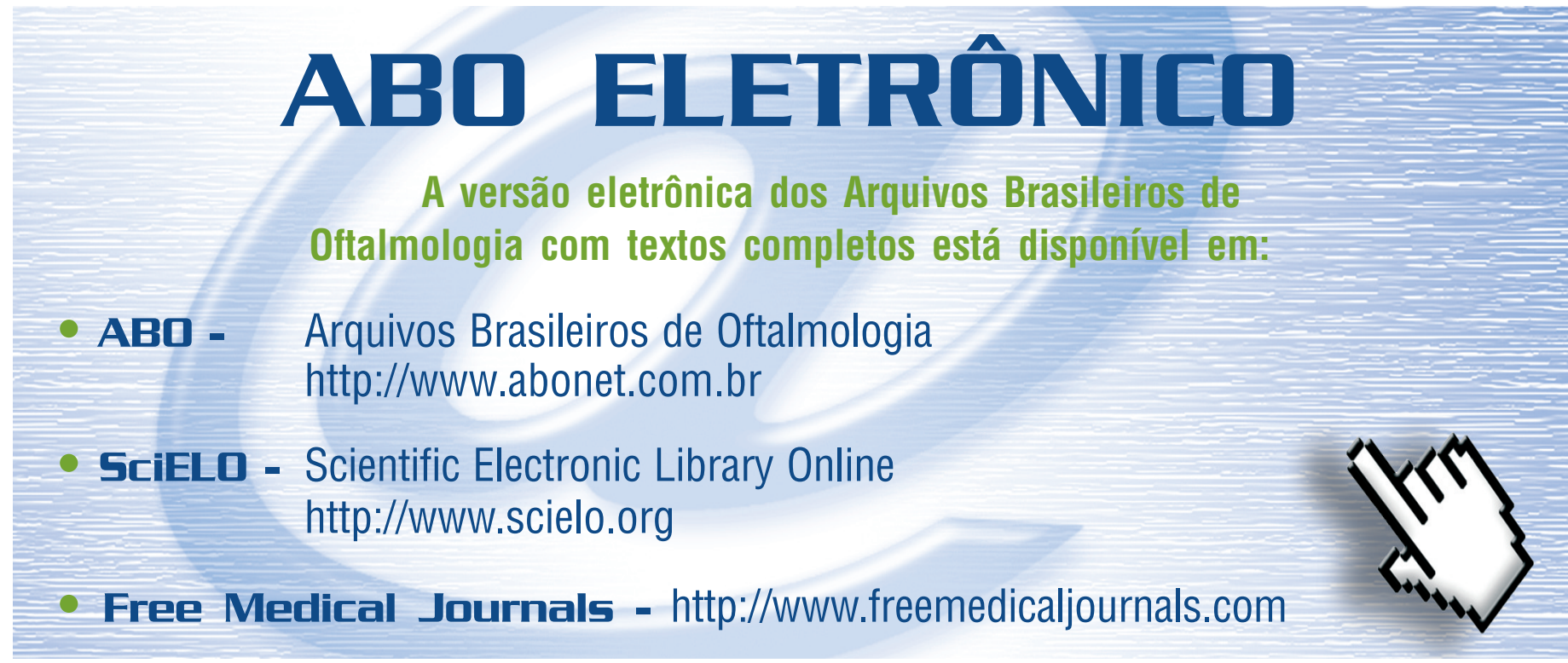

